\title{
CANONICAL SYSTEM ON ELLIPTIC CURVES
}

\author{
LUIS A. PIOVAN
}

(Communicated by Charles Pugh)

\begin{abstract}
We deduce a canonical algebraic complete integrable system using the representation of the Heisenberg group. This system is shown to have solutions equivalent to those of the rigid body motion on $\mathrm{SO}(3)$ (Euler Top).
\end{abstract}

Let $A$ be an abelian variety embedded into some projective space and $\mathscr{L}=$ $\mathscr{L}(D)$ the invertible sheaf associated with the divisor $D$ cut out by the hyperplane section at infinity. The Heisenberg group is isomorphic to $\mathscr{G}(\mathscr{L})=\{$ set of pairs $(x, \varphi)$ such that $x \in A$ and $\varphi: \mathscr{L} \approx T^{*} \mathscr{L}$ is an isomorphism\}, and, since it contains the geometric information of an abelian variety, it plays a fundamental role in the description of the equations defining abelian varieties [Mu]. This group can also be used to represent vector fields on abelian varieties. Indeed, the Wronskian with respect to the vector field $X$ on $A$ (in short, $W(f, g)=f X g-g X f)$ is invariant under the action of the Heisenberg group $[\mathrm{P}]$. This allows us to represent holomorphic vector fields on abelian varieties in a canonical fashion by means of some free parameters related to the equations defining the variety.

We realize a system by presenting it as the data $\left(A_{\alpha}, D_{\alpha}, X\right)$ where $A_{\alpha}$ is an abelian variety, $D_{\alpha}$ is a divisor on $A_{\alpha}$, and $X$ is a vector field on $A_{\alpha}$, parametrized by the integrals of the motion. Such parameters are seen to be (rationally) related to modular forms of a certain level.

The aim of this note is to provide an analysis leading to the following system of differential equations:

$$
\dot{y}_{0}=-\gamma y_{1} y_{2}, \quad \dot{y}_{1}=-\beta y_{0} y_{2}, \quad \dot{y}_{2}=-\alpha y_{0} y_{1}
$$

which defines (under certain restrictions on $\alpha, \beta, \gamma$ ) a holomorphic vector field on each elliptic curve in $\mathbb{P}^{3}$. We prove the following

Theorem. The system $(*)$ associated with the canonical family of elliptic curves in $\mathbb{P}^{3}$ and the Euler Top system (4) define in general equivalent vector fields on an elliptic curve. The linear map in $\mathbb{P}^{3}$ preserving the elliptic curves and the vector fields up to a constant is given by the map (7).

The basic technique used here is the Schrödinger representation of the Heisenberg group. This can be useful in describing algebraic complete integrable systems linearizing on higher-dimensional abelian varieties (such as those discussed

Received by the editors February 16, 1992.

1991 Mathematics Subject Classification. Primary 58F07. 
in $[A v M]$ ), in particular, in finding their canonical equations and invariants. See [Ba] for a different approach to this problem. Our analysis applied to elliptic curves leads essentially to the Euler Top. Thus, we have

Corollary. The only holomorphic vector field on the family of elliptic curves in $\mathbb{P}^{3}$ is (up to linear equivalence) given by the Euler-Arnold equations of the rigid body in $\mathrm{SO}(3)$.

\section{RePresentations of the Heisenberg GRoup $\mathscr{G}(4)$}

In the sequel we use the notation and definitions of Mumford [Mu].

The Heisenberg group $\mathscr{G}(4)$ is the set $\mathbb{C}^{*} \times K(4) \times \widehat{K(4)}, K(4) \approx \widehat{K(4)} \approx \mathbb{Z} / 4$. Its group law is

$$
(\alpha, \sigma, \chi) \cdot\left(\alpha^{\prime}, \sigma^{\prime}, \chi^{\prime}\right)-\left(\alpha \cdot \alpha^{\prime} \cdot \chi^{\prime}(\sigma), \sigma+\sigma^{\prime}, \chi+\chi^{\prime}\right) .
$$

If $V(4)$ denotes the vector space of $\mathbb{C}$-valued functions in $K(4)$, then the action of $\mathscr{G}(4)$ on this space is given by

$$
((\alpha, \sigma, \chi) \cdot f)(y)=\alpha \cdot \chi(y) \cdot f(\sigma+y) .
$$

Let $\sigma$ be the generator of $K(4)$ and $\chi$ the generator of $\widehat{K(4)}$ such that $\chi(\sigma)=i=\sqrt{-1}$. A basis for $V(4)$ has the form $X_{i}=\delta_{\sigma^{i}}, i=0,1,2,3$, where $\delta_{\sigma}\left(\sigma^{j}\right)=\delta_{i j}$, and the action of $\sigma=(1, \sigma, 1)$ and $\chi=(1,1, \chi)$ is

$$
\sigma \cdot X_{i}=X_{i-1}, \quad \chi \cdot X_{i}=(\sqrt{-1})^{i} X_{i} .
$$

Analogously, there is a "squared action" of $\mathscr{G}(4)$ on $V(8)=$ vector space of $\mathbb{C}$-valued functions on $\mathbb{Z} / 8=\left\langle\delta_{k}=\delta_{\tau}, k=0,1, \ldots, 7\right\rangle$ (see [Mu, p. 316]). This is described by the formula

$$
\left(\left(\alpha, \sigma^{u}, \chi^{v}\right) \cdot \delta_{k}\right)\left(\tau^{s}\right)=\alpha^{2} \chi(\sigma)^{v \cdot s} \delta_{k-2 u}\left(\tau^{s}\right), \quad \chi(\sigma)=\sqrt{-1} .
$$

However, instead of $X_{i}$ and $\delta_{j}$, we shall use a basis that distinguishes between odd and even sections with respect to the -1 involution $l(l f(u)=$ $f(-u))$ :

$$
\begin{aligned}
& \left\{Y_{0}=X_{0}+X_{2}, Y_{1}=X_{0}-X_{2}, Y_{2}=X_{1}+X_{3}, Y_{3}=X_{1}-X_{3}\right\} \quad \text { for } V(4) \\
& \text { and }
\end{aligned}
$$

$$
\begin{aligned}
\left\{Z_{0}\right. & =\delta_{0}+\delta_{4}, Z_{1}=\delta_{0}-\delta_{4}, Z_{2}=\delta_{2}+\delta_{6}, Z_{3}=\delta_{2}-\delta_{6}, \\
Z_{4} & \left.=\delta_{1}+\delta_{5}, Z_{5}=\delta_{1}-\delta_{5}, Z_{6}=\delta_{3}+\delta_{7}, Z_{7}=\delta_{3}-\delta_{7}\right\} \quad \text { for } V(8) .
\end{aligned}
$$

Thus, the actions of $\mathscr{G}(4)$ on $V(4)$ and $V(8)$ are described in Tables I and II.

TABLE I. Action of $\mathscr{G}(4)$ on $V(4)$.

$$
\begin{array}{ccccc} 
& Y_{0} & Y_{1} & Y_{2} & Y_{3} \\
\sigma & Y_{2} & -Y_{3} & Y_{0} & Y_{1} \\
\chi & Y_{1} & Y_{0} & i Y_{3} & i Y_{2} \\
\sigma^{2} & 1 & -1 & 1 & -1 \\
\chi^{2} & 1 & 1 & -1 & -1
\end{array}
$$


TABLE II. Action of $\mathscr{G}(4)$ on $V(8)$.

$\begin{array}{ccccccccc} & Z_{0} & Z_{1} & Z_{2} & Z_{3} & Z_{4} & Z_{5} & Z_{6} & Z_{7} \\ \sigma & Z_{2} & -Z_{3} & Z_{0} & Z_{1} & Z_{6} & -Z_{7} & Z_{4} & Z_{5} \\ \chi & 1 & 1 & -1 & -1 & i & i & -i & -i \\ \sigma^{2} & 1 & -1 & 1 & -1 & 1 & -1 & 1 & -1 \\ \chi^{2} & 1 & 1 & 1 & 1 & -1 & -1 & -1 & -1\end{array}$

\section{NORMAL EQUATIONS FOR ELLIPTIC CURVES IN $\mathbb{P}^{3}$}

For the sake of completeness we shall deduce normal equations for elliptic curves in $\mathbb{P}^{3}$.

The invertible sheaf $\mathscr{L}=\mathscr{L}(4 e)$ gives a projectively normal embedding of the elliptic curve $A$ in projective space $\mathbb{P}^{3}$ via a basis of sections $s_{i} \in \Gamma(A, \mathscr{L})$, $i=1,2,3, A \rightarrow \mathbb{P}(T(A, \mathscr{L}))$. In this case $\mathscr{L}=i^{*}(\mathscr{O}(1))$ (or, in other terms, the divisor $4 e$ is cut out by a hyperplane in $\left.\mathbb{P}^{3}\right)$.

Consider the sequence

$$
0 \rightarrow \mathscr{I}(2) \rightarrow \mathscr{O}_{\mathbb{P}^{3}}(2) \stackrel{\text { res }}{\longrightarrow} \mathscr{O}_{A}(2) \rightarrow 0
$$

where $\mathscr{I}$ is the ideal sheaf. This leads to the exact sequence

$$
0 \rightarrow \Gamma\left(\mathbb{P}^{3}, \mathscr{I}(2)\right) \rightarrow \Gamma\left(\mathbb{P}^{3}, \mathscr{O}_{\mathbb{P}^{3}}(2)\right) \rightarrow \Gamma\left(A, \mathscr{O}_{A}(2)\right) \rightarrow 0 .
$$

But $\Gamma\left(\mathbb{P}^{3}, \mathscr{O}_{\mathbb{P}^{3}}(2)\right) \cong S^{2}(\Gamma(A, \mathscr{L}))$ is the space of homogeneous polynomials of degree 2 in $s_{0}, s_{1}, s_{2}, s_{3}$ (or $4 \times 4$ symmetric matrices). Therefore,

$$
\operatorname{dim} \Gamma\left(\mathbb{P}^{3}, O_{\mathbb{P}^{3}}(2)\right)=10
$$

and

$$
\operatorname{dim} \Gamma\left(A, \mathscr{O}_{A}(2)\right)=\operatorname{dim} \Gamma\left(A, \mathscr{L}^{\otimes 2}\right)=2 \operatorname{dim} \Gamma(A, \mathscr{L})=8 .
$$

Hence, there are two linearly independent quadratic equations defining the embedded curve.

Since $\mathscr{L}$ is very ample, the second arrow of (1) amounts to the canonical map $S^{2}(\Gamma(A, \mathscr{L})) \rightarrow \Gamma\left(A, \mathscr{L}^{\otimes 2}\right)$ given by tensoring sections. Now, a symmetric theta structure for $\mathscr{L}[\mathrm{Mu}$, pp. 317-318] induces isomorphisms (unique up to scalar multiple) $V(4) \cong \Gamma(A, \mathscr{L}), V(8) \cong \Gamma\left(A, \mathscr{L}^{\oplus 2}\right)$, and we get a sequence of $\mathscr{G}(4)$-modules

$$
0 \rightarrow K \rightarrow S^{2}(V(4)) \rightarrow V(8) \rightarrow 0
$$

which is isomorphic to (1).

On the space of $4 \times 4$ symmetric matrices, the action of $\mathscr{G}(4)$ leaves invariant the following spaces marked with $A, B, C, D$ :

$\begin{array}{lllll} & Y_{0} & Y_{1} & Y_{2} & Y_{3} \\ Y_{0} & A & B & C & D \\ Y_{1} & & A & D & C \\ Y_{2} & & & A & B \\ Y_{3} & & & & A\end{array}$

The space $K \cong \Gamma\left(\mathbb{P}^{3}, \mathscr{I}(2)\right)$ cannot be contained in $B, D$, or $C$. If so, $K$ must be either $B$ or $C$ or $D$. But such quadrics cannot be zero on an 
TABLE III

\begin{tabular}{ccrrrc} 
& $Y_{0}$ & $Y_{1}$ & $Y_{2}$ & $Y_{3}$ & invariant line \\
\hline 1 & 1 & 1 & 1 & 1 & - \\
$\sigma_{1}=\sigma^{2}$ & 1 & -1 & 1 & -1 & $\left\{Y_{1}=0, Y_{3}=0\right\}$ \\
$\chi_{1}=\chi^{2}$ & 1 & 1 & -1 & -1 & $\left\{Y_{2}=0, Y_{3}=0\right\}$ \\
$\sigma_{1} \cdot \chi_{1}$ & 1 & -1 & -1 & 1 & $\left\{Y_{1}=0, Y_{2}=0\right\}$
\end{tabular}

elliptic curve, or else they contain lines which are fixed under the action of the 2-torsion translation group (Table III).

Therefore $\Gamma\left(\mathbb{P}^{3}, \mathscr{J}(2)\right)$ is contained in the four-dimensional space of diagonal quadrics.

On the quadratics, polynomials $\sigma$ and $\chi$ act as follows:

$$
\begin{array}{ccccccccc} 
& Y_{0}^{2} & Y_{1}^{2} & Y_{2}^{2} & Y_{3}^{2} & Y_{0}^{2}+Y_{1}^{2} & Y_{2}^{2}+Y_{3}^{2} & Y_{0}^{2}-Y_{1}^{2} & Y_{2}^{2}-Y_{3}^{2} \\
\sigma & Y_{2}^{2} & Y_{3}^{2} & Y_{0}^{2} & Y_{1}^{2} & Y_{2}^{2}+Y_{3}^{2} & Y_{0}^{2}+Y_{1}^{2} & Y_{2}^{2}-Y_{3}^{2} & Y_{0}^{2}-Y_{1}^{2} \\
\chi & Y_{1}^{2} & Y_{0}^{2} & -Y_{3}^{2} & -Y_{2}^{2} & 1 & -1 & -1 & 1
\end{array}
$$

and the space of diagonal quadrics decomposes into two isomorphic irreducible representations of dimension two. Generators are

$$
q_{1}=a\left(Y_{0}^{2}+Y_{1}^{2}\right)+b\left(Y_{2}^{2}-Y_{3}^{2}\right), \quad q_{2}=b\left(Y_{0}^{2}-Y_{1}^{2}\right)+a\left(Y_{2}^{2}+Y_{3}^{2}\right) .
$$

The space of diagonal quadrics contains nontrivial modules generated by rank2 quadrics, but such a module does not give equations for an elliptic curve. Therefore, the space $\Gamma\left(\mathbb{P}^{3}, \mathscr{I}(2)\right)$ is generated by $q_{1}$ and $q_{2}$, and the curve is completely characterized by the parameter $\lambda=b / a$.

\section{The CANONICAL Vector FIELD}

We want to determine the equations of a globally defined vector field $X$ in $\mathbb{P}^{3}$ whose flow is linear on each curve.

If $X$ is a vector field on an abelian variety $A$ and $s$ and $t$ are sections of the invertible sheaf $\mathscr{L}$, we define the Wronskian along $X$ by

$$
W_{X}(s, t)=W_{X}\left(\frac{f_{\alpha}}{h_{\alpha}}, \frac{g_{\alpha}}{h_{\alpha}}\right)=\frac{f_{\alpha} \cdot X g_{\alpha}-g_{\alpha} \cdot X f_{\alpha}}{h_{\alpha}^{2}} \text { on } U_{\alpha}
$$

where $\left\{\left(U_{\alpha}, h_{\alpha}\right)\right\}$ is the local data of a divisor $D$ such that $\mathscr{L}=\mathscr{L}(D)$. This gives a well-defined pair

$$
W_{X}: \Gamma(A, \mathscr{L}) \otimes \Gamma(A, \mathscr{L}) \rightarrow \Gamma\left(A, \mathscr{L}^{\otimes 2}\right)
$$

which has the following properties:

(1) $\imath^{*} W_{X}(s, t)=-W_{X}\left(t^{*} s, t^{*} t\right), l=$ the $(-1)$ involution (on sections $l^{*} s(u)$ $=s(-u))$.

(2) $W_{X}$ is invariant under the action of the Heisenberg group. Namely, if $(x, \varphi) \in \mathscr{G}(\mathscr{L}),\left(x, \varphi^{\otimes 2}\right) \in \mathscr{G}\left(\mathscr{L}^{\otimes 2}\right)$, and $U_{(x, \varphi)}: \Gamma(A, \mathscr{L}) \rightarrow \Gamma(A, \mathscr{L})$ is the action of $(x, \varphi): U_{(x, \varphi)}(s)=T_{-x}^{*}(\varphi(s))$, then

$$
U_{\left(x, \varphi^{\otimes 2}\right)} \cdot W_{X}(s, t)=W_{X}\left(U_{(x, \varphi)} \cdot s, U_{(x, \varphi)} \cdot t\right) .
$$

We use this invariance property and Tables I and II to get the Wronskian matrix 
$\left(W_{X}\left(Y_{i}, Y_{j}\right)\right)$. Here $\alpha, \beta, \gamma$ are free parameters:

$$
\begin{aligned}
& \left(W_{X}\left(Y_{i}, Y_{j}\right)\right) \\
& \quad=\left[\begin{array}{cccc}
0 & -\alpha \cdot Z_{3} & \beta \cdot\left(Z_{4}-Z_{6}\right) & \gamma \cdot\left(Z_{5}-Z_{7}\right) \\
\alpha \cdot Z_{3} & 0 & \gamma \cdot\left(Z_{5}+Z_{7}\right) & \beta \cdot\left(Z_{4}+Z_{6}\right) \\
-\beta \cdot\left(Z_{4}-Z_{6}\right) & -\gamma \cdot\left(Z_{5}+Z_{7}\right) & 0 & \alpha \cdot Z_{1} \\
-\gamma \cdot\left(Z_{5}-Z_{7}\right) & -\beta \cdot\left(Z_{4}+Z_{6}\right) & -\alpha \cdot Z_{1} & 0
\end{array}\right] .
\end{aligned}
$$

Furthermore, we can use the multiplication formula [Mu, p. 330] to obtain the description

$$
\left(W_{X}\left(Y_{i}, Y_{j}\right)\right)=\left[\begin{array}{cccc}
0 & -\alpha Y_{2} Y_{3} & \beta Y_{1} Y_{3} & \gamma Y_{1} Y_{2} \\
\alpha Y_{2} Y_{3} & 0 & \gamma Y_{0} Y_{3} & \beta Y_{0} Y_{2} \\
-\beta Y_{1} Y_{3} & -\gamma Y_{0} Y_{3} & 0 & \alpha Y_{0} Y_{1} \\
-\gamma Y_{1} Y_{2} & -\beta Y_{0} Y_{2} & -\alpha Y_{0} Y_{1} & 0
\end{array}\right]
$$

The equations of the vector field $X$ can be obtained from this description of $W$. In the coordinates $y_{i}=Y_{i} / Y_{3}$ we have

$$
X\left(\frac{Y_{i}}{Y_{3}}\right)=\frac{Y_{3} X Y_{i}-Y_{i} X Y_{3}}{Y_{3}^{2}}=\frac{W\left(Y_{3}, Y_{i}\right)}{Y_{3}^{2}}, \quad\left\{\begin{array}{l}
\dot{y}_{0}=-\gamma y_{1} y_{2}, \\
\dot{y}_{1}=-\beta y_{0} y_{2}, \\
\dot{y}_{2}=-\alpha y_{0} y_{1}
\end{array}\right.
$$

To determine the integrals, we look for quadratic polynomials in $y_{i}, i=$ $1,2,3$. These are the forms killed by the vector field $X$. There is a basis of such forms invariant under the subgroup of $\mathscr{G}(\mathscr{L})$ that fixes the chosen section $Y_{3}=0$. The action of this group is given by:

$$
\begin{array}{cccc} 
& y_{0} & y_{1} & y_{2} \\
\sigma^{2} & -1 & 1 & -1 \\
\chi^{2} & -1 & -1 & 1
\end{array}
$$

So, such quadratic integrals are $a y_{0}^{2}+b y_{1}^{2}+c y_{2}^{2}$, with $a \alpha+b \beta+c \gamma=0$ the relations in the parameters. Thus, according to (2), a basis of the equations of the curve is $a_{i} y_{0}^{2}+b_{i} y_{1}^{2}+c_{1} y_{2}^{2}=h_{i}, i=1,2$, for some constants $h_{i}$. For a vector field (3) there are Laurent solutions (in terms of the evolution parameter $t)$ about the divisor at infinity:

$$
\begin{aligned}
& y_{0}=\frac{\delta_{1}}{\sqrt{\beta \alpha}} \frac{1}{t}\left(1-(u+v) t^{2}-\frac{1}{10}\left(4 u v+(u+v)^{2}\right) t^{4}+\cdots\right), \\
& y_{1}=\frac{\delta_{2}}{\sqrt{\alpha \gamma}} \frac{1}{t}\left(1+u t^{2}+\frac{1}{10}\left(4 v(u+v)-u^{2}\right) t^{4}+\cdots\right), \\
& y_{2}=\frac{\delta_{1} \delta_{2}}{\sqrt{\gamma \beta}} \frac{1}{t}\left(1+v t^{2}+\frac{1}{10}\left(4 u(u+v)-v^{2}\right) t^{4}+\cdots\right),
\end{aligned}
$$

where $\delta_{1}^{2}=\delta_{2}^{2}=1$,

$$
\begin{aligned}
& u=\frac{1}{6}\left(\left(h_{2} a_{1}-h_{1} a_{2}\right) \gamma+\left(h_{1} c_{2}-h_{2} c_{1}\right) \alpha\right), \\
& v=\frac{1}{6}\left(\left(h_{1} a_{2}-h_{2} a_{1}\right) \gamma+\left(h_{2} b_{1}-h_{1} b_{2}\right) \beta\right) .
\end{aligned}
$$


The divisor $D$ at infinity (hyperplane section $Y_{3}=0$ ) is the formal sum of the points

$$
Q\left(\delta_{1}, \delta_{2}\right)=\left[\frac{\delta_{1}}{\sqrt{\beta \alpha}}: \frac{\delta_{2}}{\sqrt{\alpha \gamma}}: \frac{\delta_{1} \delta_{2}}{\sqrt{\gamma \beta}}: 0\right], \quad \delta_{1}^{2}=\delta_{2}^{2}=1 .
$$

It coincides with the four points fixed under the involution $l$. So the support of $D$ is precisely a two-torsion translation orbit for the origin $e=Q(1,1)$.

\section{THE EULER TOP SYSTEM AS RELATED TO THE CANONICAL SYSTEM}

The complexified Euler top system is described as the system of differential equations $[\mathrm{Ar}]$

$$
\left\{\begin{array}{l}
\dot{z}_{1}=\left(\lambda_{3}-\lambda_{2}\right) z_{2} z_{3}, \\
\dot{z}_{2}=\left(\lambda_{1}-\lambda_{3}\right) z_{1} z_{3}, \\
\dot{z}_{3}=\left(\lambda_{2}-\lambda_{1}\right) z_{1} z_{2},
\end{array} \quad\left(z_{1}, z_{2}, z_{3}\right) \in \mathbb{C}^{3} .\right.
$$

The invariant curves

$$
p_{1}: z_{1}^{2}+z_{2}^{2}=z_{3}^{2}=0, \quad p_{2}: \lambda_{1} z_{1}^{2}+\lambda_{2} z_{2}^{2}+\lambda_{3} z_{3}^{2}-h=0
$$

are the affine part of elliptic curves obtained by adding four points at infinity (a divisor $\left.D^{\prime}\right)$. Moreover, the invertible sheaf $\mathscr{L}=\mathscr{L}\left(D^{\prime}\right) \approx \mathscr{L}\left(4 e^{\prime}\right)$ determines a projectively normal embedding via the functions $\left\{1, z_{1}, z_{2}, z_{3}\right\}$ which blow up at $D^{\prime}$. Around $D^{\prime}$ we have the Taylor expansions in terms of $t=$ time evolution parameter:

$$
\begin{array}{rlrl}
z_{1} & =\frac{\varepsilon_{1}}{\sqrt{\alpha_{2} \alpha_{3}}} \frac{1}{t}\left(1-\left(u^{\prime}+v^{\prime}\right) t^{2}+\cdots\right), & \varepsilon_{1}^{2} & =\varepsilon_{2}^{2}=1, \alpha_{1}=\lambda_{3}-\lambda_{2}, \\
z_{2} & =\frac{\varepsilon_{2}}{\sqrt{\alpha_{3} \alpha_{1}}} \frac{1}{t}\left(1+u^{\prime} t^{2}+\cdots\right), & \alpha_{2}=\lambda_{1}-\lambda_{3}, \alpha_{3}=\lambda_{2}-\lambda_{1} \\
z_{3}=\frac{\varepsilon_{1} \varepsilon_{2}}{\sqrt{\alpha_{1} \alpha_{2}}} \frac{1}{t}\left(1+v^{\prime} t^{2}+\cdots\right), & v^{\prime}=\frac{1}{6}\left(\left(\lambda_{3}-h\right) \alpha_{3}+\left(h-\lambda_{1}\right) \alpha_{1}\right) \\
6 & \left(\left(h-\lambda_{2}\right) \alpha_{2}+\left(\lambda_{1}-h\right) \alpha_{1}\right) .
\end{array}
$$

Using the invariance of the vector field under translations one gets involutions

$$
\begin{aligned}
& \sigma_{1}^{\prime}:\left(z_{1}, z_{2}, z_{3}\right) \rightarrow\left(-z_{1}, z_{2},-z_{3}\right), \\
& \tau_{1}^{\prime}:\left(z_{1}, z_{2}, z_{3}\right) \rightarrow\left(-z_{1},-z_{2}, z_{3}\right), \\
& \sigma_{1}^{\prime} \tau_{1}^{\prime}:\left(z_{1}, z_{2}, z_{3}\right) \rightarrow\left(z_{1},-z_{2},-z_{3}\right)
\end{aligned}
$$

which amount to translations by $\frac{1}{2}$-periods.

The involution flipping the sign of the vector field

$$
l:\left(z_{1}, z_{2}, z_{3}\right) \rightarrow\left(-z_{1},-z_{2},-z_{3}\right)
$$

fixes precisely the points of the divisor $D^{\prime}$ :

$$
P\left(\varepsilon_{1}, \varepsilon_{2}\right)=\left[0: \frac{\varepsilon_{1}}{\sqrt{\alpha_{2} \alpha_{3}}}: \frac{\varepsilon_{2}}{\sqrt{\alpha_{3} \alpha_{1}}}: \frac{\varepsilon_{1} \varepsilon_{2}}{\sqrt{\alpha_{1} \alpha_{2}}}\right], \quad \varepsilon_{1}^{2}-\varepsilon_{2}^{2}=1 .
$$

Any of these points can be chosen as origin. Let us pick $e^{\prime}=P(1,1)$ and consider the $2: 1$ map $\varphi^{\prime}: A \rightarrow \mathbb{P}^{1}$ via the basis of sections of $\mathscr{L}\left(2 e^{\prime}\right)$ : $\left\{1, f=\left(\sqrt{\alpha_{2} \alpha_{3}} z_{1}+\sqrt{\alpha_{1} \alpha_{3}} z_{2}\right)\left(\sqrt{\alpha_{2} \alpha_{3}} z_{1}+\sqrt{\alpha_{1} \alpha_{2}} z_{3}\right)\right\}$. Analogously, the basis 
of sections of $\mathscr{L}(2 e)$ given by 1 and $g=\left(\sqrt{\beta \alpha} y_{0}+\sqrt{\gamma \alpha} y_{1}\right)\left(\sqrt{\beta \alpha} y_{0}+\sqrt{\gamma \beta} y_{2}\right)$ determines a $2: 1$ cover $\varphi: A \rightarrow \mathbb{P}^{1}$ branched over four points, the image of the four $\frac{1}{2}$-periods. In order to relate the Euler Top to our canonical system we want to describe a map that preserves the chosen origins $e \leftrightarrow e^{\prime}$ and $\frac{1}{2}$ periods translations via the isomorphism $\sigma \leftrightarrow \sigma \quad \chi \leftrightarrow \tau$. This induces a linear transformation of $\mathbb{P}^{1}$ which goes as follows:

$$
\begin{aligned}
& f\left(e^{\prime}\right)=f(P(1,1))=\infty, \\
& f\left(\sigma_{1}^{\prime} e^{\prime}\right)=f(P(-1,1))=-2\left(2 u^{\prime}+v^{\prime}\right)=\left(\lambda_{2}-\lambda_{1}\right)\left(h-\lambda_{3}\right), \\
& f\left(\tau_{1}^{\prime} e^{\prime}\right)=f(P(-1,-1))=-2\left(u^{\prime}+2 v^{\prime}\right)=\left(\lambda_{1}-\lambda_{3}\right)\left(\lambda_{2}-h\right), \\
& f\left(\sigma_{1}^{\prime} \tau_{1}^{\prime} e^{\prime}\right)=f(P(1,-1))=0, \\
& g(e)=g(Q(1,1))=\infty, \\
& g\left(\sigma_{1} e\right)=g(Q(-1,1))=-2(2 u+v), \\
& g\left(\chi_{1} e\right)=g(Q(-1,-1))=-2(u+2 v), \\
& g\left(\sigma_{1} \chi_{1} e\right)=g(Q(1,-1))=0 .
\end{aligned}
$$

Hence,

$$
f=\frac{2 u+v}{2 u^{\prime}+v^{\prime}} g=c \cdot g
$$

and we get the quantity

$$
\omega=\frac{\left(\lambda_{1}-\lambda_{3}\right)\left(\lambda_{2}-h\right)}{\left(\lambda_{2}-\lambda_{1}\right)\left(h-\lambda_{3}\right)}=\frac{u+2 v}{2 u+v}=\frac{u^{\prime}+2 v^{\prime}}{2 u^{\prime}+v^{\prime}} .
$$

This quantity represents the moduli of an elliptic curve $A^{\prime}$ isogenous to $A$. The linear transformation (5) extends to a linear mapping in $\mathbb{P}^{3}$. Indeed, $\left\{1, f, f^{2}, X_{e} f\right\}$ and $\left\{1, g, g^{2}, X_{c} g\right\}$ are bases of sections of $\mathscr{L}\left(4 e^{\prime}\right)$ and $\mathscr{L}(4 e)$, where $X_{e}=$ Euler vector field $=c^{1 / 2} \cdot X_{c}=c^{1 / 2}$ (canonical vector field), thus, inducing the obvious linear map. In terms of the variables $z_{i}$ and $y_{i}$ we have

$$
z_{1}=\sqrt{\frac{\beta \alpha c^{3}}{\alpha_{2} \alpha_{3}}} y_{0}, \quad z_{2}=\sqrt{\frac{\alpha \gamma c^{3}}{\alpha_{3} \alpha_{1}}} y_{1}, \quad z_{3}=\sqrt{\frac{\gamma \beta c^{3}}{\alpha_{1} \alpha_{2}}} y_{2}
$$

\section{REFERENCES}

[AvM] M. Adler and P. van Moerbeke, Completely integrable systems-A systematic approach towards solving integrable systems, preprint.

[Ar] V. Arnold, Mathematical methods of classical mechanics, Springer-Verlag, New York, 1978.

[Ba] W. Barth, Affine parts of abelian surfaces as complete intersections of four quadrics, Math. Ann. 278 (1987), 117-131.

[Mu] D. Mumford, On the equations defining Abelian varieties, I, II, Invent. Math. 1 (1966), 287-354; 2, 3 (1967), 75-135, 215-244.

[P] L. Piovan, Algebraically completely integrable systems and Kummer varieties, Math. Ann. 290 (1991), 349-403.

Departamento de Matematica, Universidad Nacional del Sur, 8000 Bahia Blanca, ARgentina

E-mail address: impiovan@arcriba.edu.ar 